\title{
Correlación entre estadíos de calcificación de caninos y segundas premolares mandibulares con la curva de crecimiento puberal maxilar y mandibular
}

Valverde R, Adriazola M, Meneses A. Correlación entre estadíos de calcificación de caninos y segundas premolares mandibulares con la curva de crecimiento puberal maxilar y mandibular. Rev Estomatol Herediana 2004;14(1-2) : 12 - 17.

\section{RESUMEN}

El propósito del presente estudio fue determinar el grado de correlación entre los estadios de calcificación dentaria según Demirjian para caninos y segundas premolares mandibulares, y la curva de crecimiento puberal maxilar y mandibular según Fishman. La muestra consistió en 183 pares de radiografías, panorámicas y carpales, 100 de mujeres (54.64\%) y 83 de hombres, (45.36\%), tomadas el mismo día. La muestra, se subagrupó según sexo y lado a que pertenecía cada diente analizado. Se utilizaron la prueba de correlación de Pearson y tablas de distribución de frecuencia. Las correlaciones encontradas fueron altamente significativas en todos los casos; la correlación más alta respecto a Fishman se dio con el segundo premolar derecho (0.882), y la más baja con el canino derecho (0.832), la más alta correlación en cuanto a calcificación dentaria se dio entre los premolares de ambos lados (0.995). No se encontró dimorfismo sexual ni diferencias significativas en los resultados. El estadío G de calcificación dentaria según Demirjian, coincidió con el pico maximo de crecimiento puberal en mujeres (estadío 6 de Fishman) en el $86.48 \%$ de los casos, y en hombres (estadío 7 de Fishman) en el $98.21 \%$ de los casos. Se concluye que existe correlación altamente significativa entre la curva de crecimiento puberal y los estadíos de calcificación dentaria.

Palabras clave: CALCIFICACIÓN DE DIENTES / ARCADA OSEODENTARIA. crecimiento $\&$ desarrollo.

Correlation between mandibular cuspids and second bicuspids calcification and the puberal growth curve of the maxilla and mandible ABSTRACT

The present study intends to determine the degree of correlation between the Demirjian Stages of Calcification of mandibular cuspids and second bicuspids and the maxillary and mandibular Fishman Puberal Growth Curve. The sample calculated at a level of significance of $99.99 \%$ $(\mathrm{p}<0.001)$ consisted of double sets of panoramic and wrist radiographs (100 females, 54.64\% and 83 males $45.36 \%$ ) taken the same day. The sample was subdivided according to sex and the side to which each tooth annalysed belonged. Pearson correlation test and Tables of Frecuency Distribution were used. The correlation found was of a high significance in all cases, with the higher Fishman correlation found in the right second bicuspid (0.882) and the lowest correlation in the right cuspid (0.832). The higher correlation due to dental calcification was found between the bicuspids of both sides (0.995). No sexual dimorphism or significative differences were found in our results. Demirjian's "G" calcification stage coincided with the female PHV (stage 6 of Fishman) in $86.48 \%$ of the cases, and for males (stage 7 of Fishman) in $98.21 \%$. We conclude that there is a high significance correlation between the stages of calcification of mandibular cuspids and second bicuspids and the maxillary mandibular puberal growth curve.

Keywords: TOOTH CALCIFICATION / JAW. growth \& development.
Renzo Valverde Montalva ${ }^{1}$ Manuel Adriazola Pando' Abraham Meneses López ${ }^{1}$

'Docente del Departamento Académico de Estomatología del Niño y del Adolescente. Facultad de Estomatología. Universidad Peruana Cayetano Heredia

\section{Introducción}

Determinar y comprender los eventos relacionados con el crecimiento y desarrollo es de singular importancia en ortodoncia, la maduración esquelética tiene influencia en el diagnóstico, plan de tratamiento, resultados y pronóstico del tratamiento $(1,2)$.

La osificación de los huesos de la mano y muñeca, son indicadores confiables y accesibles para determinar, mediante radiografías carpales, los Estadios de Maduración Esquelética (EME), permitiéndonos ubicar al pacien- te en la curva de incremento estatural puberal Adolescente. Esta curva consta de cuatro partes: inicio de la curva de crecimiento puberal, aceleración del crecimiento puberal, Pico Máximo de Crecimiento Puberal (PMCP), y final del crecimiento puberal (1-9). 
En promedio el PMCP tiene lugar entre los 12 y 17 años, donde se observa un crecimiento rápido de todos los huesos. En las niñas, esto ocurre entre los 10 y 12 años y en niños entre los 12 y 14 años. El final del crecimiento tiene lugar en niñas alrededor de los 15 años y en niños cerca a los 17 años $(9,10)$.

Las decisiones tomadas para el uso de fuerzas extraorales, aparatos miofuncionales, y procedimientos quirúrgicos, son tomadas teniendo en cuenta el grado de maduración esquelética del individuo. Así, determinar el estadio de crecimiento puberal es importante en aquellas maloclusiones en que los componentes basales están afectados. Pancherz y Hagg (11), demuestran que el tratamiento con aparatos funcionales y arcos de tracción extraoral pudo redireccionar el crecimiento de las bases óseas durante el pico de crecimiento, mientras que en tratamientos posteriores al PMCP se observan sólo movimientos ortodóncicos, es decir, que durante el PMCP las posibilidades de tratamiento ortopédico son mayores, mientras que fuera del pico sólo es posible realizar compensaciones dentarias y las posibilidades ortopédicas son menores. En relación a fuerzas ortopédicas extraorales, Kopecky y Fishman (12), demostraron que los efectos más significativos se producen durante el PMCP, asimismo, comprobaron que la edad cronológica y la erupción dentaria no se relacionan de manera alguna con este acontecimiento. La determinación del PMCP, si este evento ya tuvo lugar, o cuanto falta para que se manifieste, es de suma importancia en algunos casos límite, casos con posible necesidad de extracciones, en casos con necesidad de aparatología ortopédica o quirúrgica, o en la prognosis de discrepancias esqueléticas Clase II y Clase III (13). Nanda (14), Bjork y Helm (15), Hagg (3-6), Fishman (1,2), Kopecky y Fishman (12), Bergensen (8), Bambha (13), entre otros, han establecido que el patrón de crecimiento y desarrollo facial es similar al del crecimiento esquelético general, y el PMCP de las estructuras cráneofaciales se da entre 6 y 8 meses después que el PMCP estatural.
Se ha demostrado la confiabilidad de las radiografías carpales para determinar el PMCP, pero existen otras propuestas para identificar el grado de maduración de los individuos como son, la edad cronológica, edad dentaria, irrupción dentaria, aparición de caracteres secundarios como menarquia, cambio de voz, etc (3-7). Diferentes parámetros han sido estudiados para determinar la edad dentaria; por lo general las piezas dentarias son visibles en la cavidad oral cuando tres cuartos de raíz están formadas, sin embargo, los estudios muestran una pobre correlación entre erupción dentaria y pico de crecimiento. Existen reportes de correlación entre estadios de calcificación dentaria y maduración esquelética, los estudios al respecto sugieren que la formación radicular es un indicador de maduración más confiable que la erupción dentaria; algunos trabajos sugieren incluso que el mejor momento para emplear una tracción extraoral es cuando el segundo premolar mandibular tiene dos tercios de raíz formada. Hoy es aceptado, que la erupción dentaria es un indicador más variable que los indicadores de maduración esquelética y que los estadios de calcificación dentaria (16-18).

Sierra (16), evaluó niños de ambos sexos basándose en el atlas elaborado por Greulich y Pyle en 1959 para determinar el estadio de maduración y en los estadios de calcificación dentaria propuestos por Nolla (18), concluyó que existe correlación significativa entre ambos acontecimientos, siendo la correlación más fuerte a nivel del canino mandibular.

Coutinho y Buschang (17), mostraron en niños y niñas norteamericanos una alta correlación entre los estadíos de calcificación de caninos mandibulares propuestos por Demirjian y el pico de crecimiento puberal según los atlas de Greulich y Pyle, los niños mostraron estadio $\mathrm{G}$ para el canino en presencia del sesamoideo (81\%), capeamiento de la tercera falange media (77\%), y capeamiento de la quinta falange proximal (87\%), de esto concluyen que el estadio $\mathrm{G}$ para el canino tiene lugar aproximadamente 0.4 años antes del pico de crecimiento para las niñas y 1.3 años antes para los niños.

Chertkow y Fatti (19), en sujetos caucásicos (93 niñas y 47 niños) que no habían sido tratados ortodóncicamente, el estudio siguió parámetros similares al presentado anteriormente llegando a los mismos resultados, los autores recomiendan el utilizar el canino mandibular como parámetro de evaluación del pico de maduración esquelética puberal.

Chertkow (20), compara pacientes de raza blanca y negra que no habían recibido tratamiento alguno, encuentra que el estadio G según Demirjian para el canino mandibular del lado izquierdo se correlacionaba con el pico de crecimiento para los parámetros de Tanner y Whitehouse.

Estudios hechos en la India por Gupta (21), señalan que la aparición temprana del sesamoideo cubital es considerado el indicador más confiable y el más utilizado para determinar el pico de crecimiento puberal. Sus resultados mostraron correlación significativa entre el estadio de calcificación $\mathrm{G}$ en caninos y la aparición del hueso sesamoideo en niñas, recomendando estos indicadores para determinar el pico de crecimiento puberal en la práctica odontológica, sin embargo estos resultados no son aplicables a niños debido a que al momento de aparición del sesamoideo cubital, el cierre apical ya era manifiesto.

\section{Evaluación carpal de maduración esquelética, según Fishman (1).}

El sistema utiliza el pulgar, el tercer dedo, quinto dedo y el radio, en donde se toman seis lugares anatómicos (Fig. 1).

La osificación progresa, pudiéndose reconocer cuatro eventos, tomados como indicadores: ensanchamiento epifisiario en las falanges, la osificación del sesamoideo abductor del pulgar, el cubrimiento de las diáfisis por sus respectivas epífisis, y la fusión de estas con sus diáfisis. Fishman señala once Estadios de Maduración Esquelética (EMEs) en la curva de crecimiento puberal. La secuencia de ocurrencia de los once indicadores es excepcionalmente estable. El estudio original, sólo 
reportó tres desviaciones en más de dos mil observaciones, y éstas no afectaron las interpretaciones (Fig. 1).

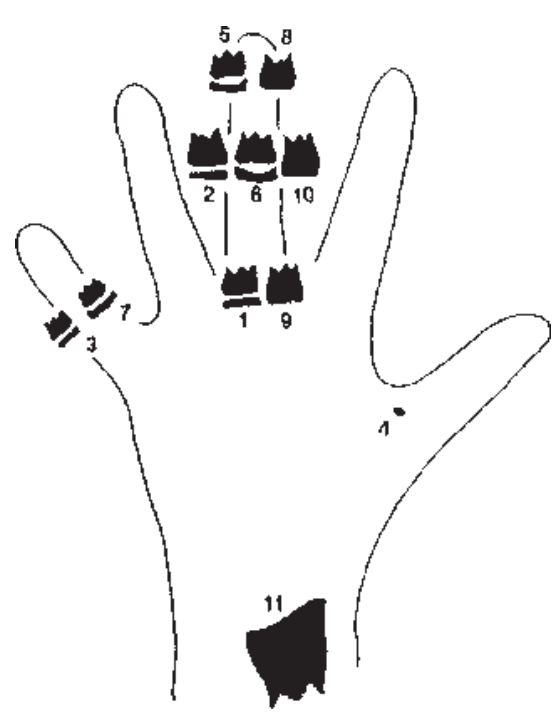

Fig. 1. EMEs: ancho de la epífisis igual al de la diáfisis: EME 1. Tercer dedo falange proximal, EME 2. Tercer dedo falange media, EME 3. Quinto dedo falange media. Osificación del sesamoideo abductor del pulgar: EME 4. Cubrimiento de las diáfisis por las epífisis: EME 5. Tercer dedo falange distal, EME 6. Tercer dedo falange media, EME 7. Quinto dedo falange media. Fusión de epífisis y diáfisis: EME 8. Tercer dedo falange distal, EME 9. Tercer dedo falange proximal, EME10. Tercer dedo falange media, EME11. Radio.

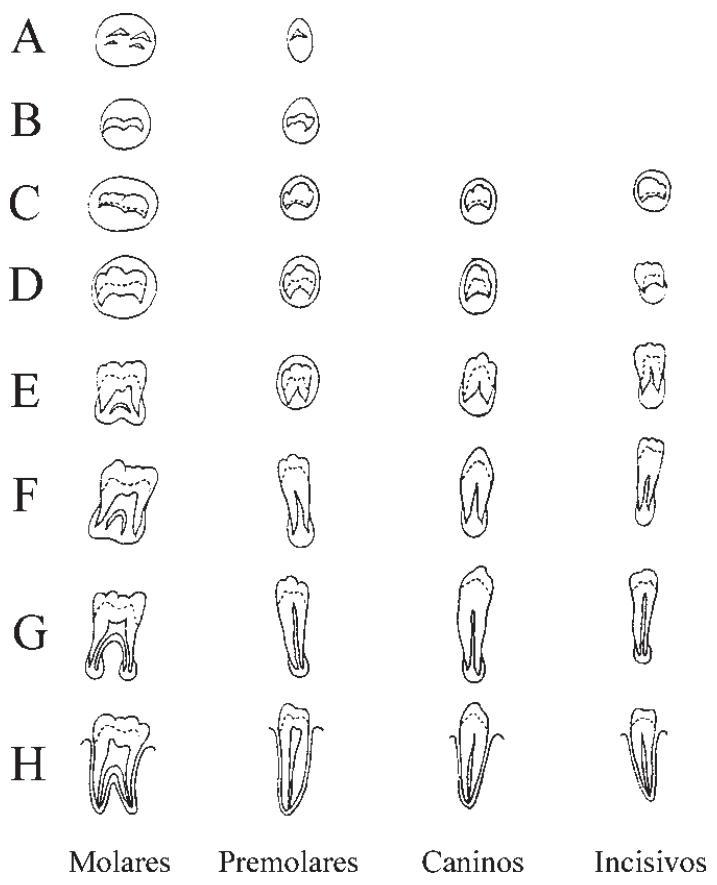

Fig. 2. Representación gráfica de los Estadíos de Calcificación Dentaria según Demirjian (22).

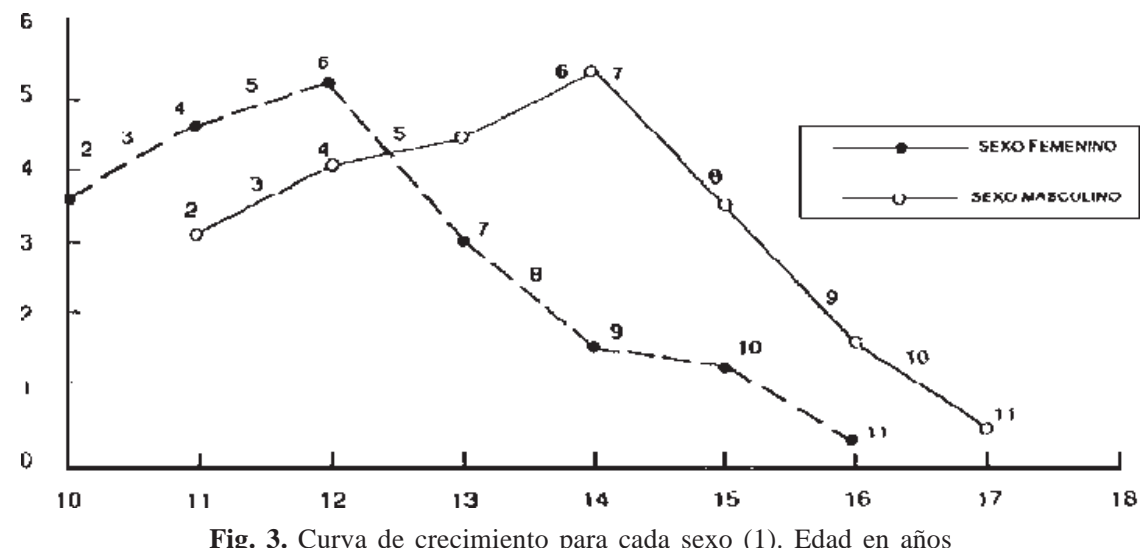

mara pulpar, y cierre apical. De acuerdo a estas características fueron determinados ocho estadios de calcificación nombrados de la A hasta la H (Fig. 2).

El objetivo del presente estudio fue determinar la correlación entre los estadios de calcificación dentaria según Demirjian, y la curva de crecimiento puberal maxilar y mandibular según Fishman (Fig. 3), y cual estadio de calcificación dentaria se correlaciona con el pico de crecimiento puberal. De existir alguna correlación entre dichos even- tos contaremos con una herramienta adicional para la determinación del pico de crecimiento puberal.

\section{Material y método}

El diseño del estudio fue transversal descriptivo y correlacional.

La muestra estuvo constituida por 183 pares de radiografías (panorámicas y carpales) de un total de 502 pacientes de la Clínica de Postgrado de Ortodoncia de la UPCH entre 8 y 15 años de edad.

Los estadios de maduración esquelética según Fishman y de calcificación 
dentaria según Demirjian se determinaron para cada radiografía de carpal y panorámica de acuerdo a criterios similares a los propuestos por Manson-Hing (23). El estudio se realizó en una habitación oscura y se utilizó un negatoscopio de luz fría, se examinaron sólo 10 radiografías por día. Utilizando una placa radiográfica velada se cubrió la radiografía a estudiar, a fin de evitar filtración de luz para mejorar la visualización.

De los estadíos evaluados por Demirjian (22) para caninos y premolares se consideró únicamente los estadios D, E, F, G, H, debido a que trabajos de investigación publicados anteriormente $(17,21)$ relacionan el PMCP con dichos estadios, esta coincidencia se encontró también en los resultados del estudio piloto.
Los datos fueron analizados mediante el índice de correlación de Pearson, tablas de distribución de frecuencias y rangos para determinar porcentajes, y la prueba de chi-cuadrado sólo para dimorfismo sexual.

\section{Resultados y discusión}

En 183 sujetos estudiados, 100 (54.64\%) mujeres y 83 (45.36\%) hombres, se calculó la correlación entre la curva de crecimiento puberal maxilar y mandibular de Fishman y los estadios de calcificación dentaria para caninos y segundos premolares mandibulares según Demirjian a un nivel de confianza de 99.99\% ( $<$ <0.001) observándose una correlación altamente significativa (Tabla 1). Meredith (24) demostró que la correlación existente entre pico de cre-

Tabla 1. Correlación entre la curva de crecimiento puberal maxilar y mandibular de fishman y los estadios de calcificación dentaria según demirjian para caninos y segundos premolares mandibulares.

\begin{tabular}{|lccccc|}
\hline C. Pearson & Fishman & Can Der & Can Izq & 2do PM der & 2do PM izq \\
\hline Fishman & 1 & $0.832^{* *}$ & $0.842^{* *}$ & $0.882^{* *}$ & $0.876^{* *}$ \\
\hline Can Der & $\mathbf{0 . 8 3 2 * *}$ & 1 & $0.982^{* *}$ & $0.917^{* *}$ & $0.922^{* *}$ \\
\hline Can Izq & $0.842^{* *}$ & $0.982^{* *}$ & 1 & $0.923^{* *}$ & $0.923^{* *}$ \\
\hline 2do PM der & $0.882^{* *}$ & $0.917^{* *}$ & $0.923^{* *}$ & 1 & $0.995^{* *}$ \\
\hline 2do PM izq & $0.876^{* *}$ & $0.922^{* *}$ & $0.923^{* *}$ & $\mathbf{0 . 9 9 5 * *}$ & 1 \\
\hline
\end{tabular}

** El segundo premolar derecho mostró la correlación más alta con la curva de crecimiento puberal maxilar y mandibular de Fishman (en amarillo) y la menor el canino derecho, sin embargo todas las correlaciones eran altamente significativas. La correlación más alta entre estadios de calcificación (en rojo) se dio entre los segundos premolares(n: 183, p<0.001).

Tabla 2. Distribución de frecuencias de los estadios de fishman en cada estadio de calcificación para caninos y segundos premolares mandibulares derechos e izquierdos, sexo femenino.

\begin{tabular}{|c|c|c|c|c|c|}
\hline FEMENINO & ESTADÍO "D" & ESTADÍO "E" & ESTADÍO "F" & ESTADÍO "G" & ESTADÍO "H" \\
\hline FISHMAN 1 & & 2 & 2 & & \\
\hline FISHMAN 2 & 4 & 16 & 8 & & \\
\hline FISHMAN 3 & 5 & 42 & 9 & & \\
\hline FISHMAN 4 & & & 12 & & \\
\hline FISHMAN 5 & & & & 4 & \\
\hline FISHMAN6 & & & 15 & 128 & 5 \\
\hline FISHMAN 7 & & & & 38 & 2 \\
\hline FISHMAN8 & & & & 4 & 32 \\
\hline FISHMAN9 & & & 4 & 16 & 20 \\
\hline FISHMAN 10 & & & & 2 & 10 \\
\hline
\end{tabular}

En amarillo, concordancia entre los estadios previos al PMCP o inicio de la curva de crecimiento puberal y los estadios de Demirjian previos al G (96.15\%). En rojo, concordancia entre el estadio correspondiente a PMCP para mujeres (Fishman 6) y el estadio $G$ de Demirjian (86.48\%). En azul, concordancia entre los estadios posteriores al PMCP o fase de descenso de la curva y el estadio $H$ de Demirjian (50.00\%), $(\mathrm{p}<0.001, \mathrm{n}: 400)$ 
Tabla 3. Distribución de frecuencias de los estadios de fishman en cada estadio de calcificación para caninos y segundos premolares mandibulares derechos e izquierdos, sexo masculino.

\begin{tabular}{|c|c|c|c|c|c|}
\hline MASCULINO & ESTADÍO "D" & ESTADÍO"E" & ESTADÍO "F" & ESTADÍO "G" & ESTADÍO "H" \\
\hline FISHMAN 1 & & 2 & 2 & & \\
\hline FISHMAN 2 & 3 & 15 & 10 & 4 & \\
\hline FISHMAN 3 & & 24 & 17 & 6 & 1 \\
\hline FISHMAN 4 & & 6 & 2 & & \\
\hline FISHMAN 5 & & & 8 & 4 & \\
\hline FISHMAN 6 & & & & 49 & 3 \\
\hline FISHMAN 7 & & & 2 & 110 & \\
\hline FISHMAN 8 & & & & 2 & 18 \\
\hline FISHMAN9 & & & & & 16 \\
\hline FISHMAN 10 & & & & & 24 \\
\hline FISHMAN 11 & & & & & 8 \\
\hline
\end{tabular}

En amarillo, concordancia entre los estadios previos al PMCP o inicio de la curva de crecimiento puberal y los estadios de Demirjian previos al G (58.55\%). En verde, concordancia entre el estadio correspondiente a PMCP para mujeres (Fishman 6) y el estadio G de Demirjian(98.21\%). En azul, concordancia entre los estadios posteriores al PMCP o fase de descenso de la curva y el estadio H de Demirjian (97.05), $(\mathrm{p}<0.001, \mathrm{n}: 332)$

las que 128 (86.48\%) coinciden con el estadio $\mathrm{G}$ de calcificación dentaria según Demirjian. De 128 ocurrencias en algún estadio descendente de la curva de crecimiento (del 7 al 11), 64 (50.00\%) coinciden con el estadio $\mathrm{H}$ de calcificación dentaria según Demirjian, 60 (46.87\%) coinciden con G, (38 de las cuales corresponden al estadio 7 de Fishman), y sólo 4 (3.12\%) con F.

En la Tabla 3, observamos en el estadio 7 de Fishman (pico máximo de crecimiento puberal para el sexo masculino) 112 ocurrencias, de las que 110 (98.21\%) coinciden con el estadio G de calcificación dentaria según Demirjian. De las 100 situaciones que se encuentran en algún estadio ascendente de la curva de crecimiento (del 1 al 6), 89 (58.55\%) coinciden con estadios de calcificación dentaria anteriores al estadio G, 59 (38.81\%) coinciden con el estadio G, y 4 (2.63\%) con el estadio H. De 68 ocurrencias en algún estadio descendiente de la curva de crecimiento (del 8 al 11), 66 (97.05\%) coinciden con el estadio $\mathrm{H}$ de calcificación dentaria según Demirjian, y 2 (2.94\%) coinciden con G.

Chertkow y Fatti (19), hallan significativa correlación (77\%) entre el estadio $\mathrm{G}$ de calcificación para el canino mandibular y la calcificación del sesamoideo, en su estudio de 140 sujetos caucásicos. En otro estudio Chertkow (20), esta vez en 197 sujetos negros y blancos, encuentra que el estadio $\mathrm{G}$ para el canino mandibular del lado izquierdo se correlacionaba con el pico de crecimiento puberal (77\% en mujeres y 78\% en hombres de ambas razas). Las correlaciones halladas en ambos estudios son bastante menores a las halladas en el presente estudio, $86.48 \%$ y $98.21 \%$ de coincidencia para caninos derecho e izquierdo en el sexo femenino y masculino respectivamente (Tablas 2 y 3 ).

Gupta (21), señala que existe correlación significativa entre el estadio de Calcificación $\mathrm{G}$ de caninos y el pico de crecimiento en mujeres, pero no en hombres debido a que al momento del PCMP, el cierre apical ya se hizo manifiesto. Nuestros resultados muestran correlaciones altamente significativas para ambos sexos.

Los cambios propios de la pubertad están visiblemente relacionados a cambios hormonales básicamente andrógenos en hombres y secreciones adrenales en mujeres, según van Wagenen (26), la administración de testosterona en monos Macaca mulatta produce aceleración en la erupción y formación de raíz de los caninos.

Los estudios de Thompson (27), señalan que existe dimorfismo sexual respecto a la distribución de los estadios de calcificación dentaria, con excepción del canino mandibular. Muestra además, que el desarrollo dental en niños tiende a ser más rápido que la maduración esquelética.
Lo descrito, explicaría la razón por la que el estadio de calcificación $\mathrm{G}$ coincide con el pico de crecimiento tanto en hombres como en mujeres, siendo el pico de crecimiento femenino de más temprana aparición que el masculino.

Demirjian (28), señala que la iniciación del pico de crecimiento puberal coincide con el estadio F, el estadio G coincide con la erupción del canino en la cavidad oral y se da un año antes del pico de crecimiento puberal en hombres y cinco meses antes en mujeres. Los resultados del presente trabajo señalan que los estadios de calcificación del canino mandibular previos al G, en mujeres se relacionan con estadios de maduración esquelética anteriores al pico de crecimiento puberal pero sin particularidad alguna. Sin embargo, en hombres además de la correlación altamente significativa entre el estadio $\mathrm{G}$ y el pico de crecimiento puberal (estadio 7 de Fishman), existe correlación entre el estadio anterior al pico de crecimiento puberal (estadio 6 de Fishman) y el estadio G de calcificación dentaria (Tablas 2 y 3$)$.

Coutinho y Buschang (17), estudiaron 200 niños y 215 niñas norteamericanos hallando también una correlación altamente significativa entre el estadio $\mathrm{G}$ de calcificación del canino mandibular izquierdo y el pico de crecimiento puberal según Greulich y Pyle. Los hombres mostraron el canino en estadio G 
1.3 años antes del pico de crecimiento puberal y las mujeres 0.4 años. El presente estudio no pretende correlacionar la presencia de los estadios de calcificación con edades cronológicas, pero si con la curva de crecimiento. Los resultados, muestran correlación significativa entre el estadio $\mathrm{H}$ de caninos, y los estadios de Fishman correspondientes al descenso y final de la curva de crecimiento, $50 \%$ y $97.05 \%$ de correspondencia para mujeres y hombres respectivamente. Aunque en la literatura sin embargo, no se señala nada al respecto del final de la curva de crecimiento. Los resultados señalan además, que existe correlación significativa entre los estadios tempranos de la curva de crecimiento puberal y los estadios anteriores al G, para segundos premolares y caninos mandibulares de ambos sexos.

\section{Conclusiones}

- Se encontró correlación altamente significativa entre el estadio $\mathrm{G}$ de caninos y segundos premolares mandibulares, con el pico máximo de crecimiento puberal maxilar y mandibular en ambos sexos.

- Se encontró correlación altamente significativa entre los estadios de calcificación anteriores al G de caninos mandibulares y segundos premolares mandibulares, con los estadios iniciales de la curva de crecimiento puberal maxilar y mandibular en ambos sexos.

- Se encontró correlación altamente significativa entre el estadio de calcificación $\mathrm{H}$ de los caninos mandibulares y segundos premolares mandibulares, con los estadios finales de la curva de crecimiento puberal maxilar y mandibular en ambos sexos.

- Se encontró correlación altamente significativa entre caninos y segundos premolares mandibulares del lado derecho e izquierdo en ambos sexos. Y no se encontró dimorfismo sexual entre las correlaciones halladas.

\section{Referencias bibliográficas}

1. Fishman L. Radiographic evaluation of skeletal maturation. A clinical oriented method on hand - wrist film. Angle Orthod 1982; 52: 88 - 112.

2. Fishman L. Maturational patterns and prediction during adolescence. Angle Orthod 1987; 63(4) 178 - 93.

3. Hägg U. Taranger J. Maturation indicators and the pubertal growth spurt. Am J. Orthod. 1982; 82(4): 299 - 309.

4. Hägg U. Taranger J. Menarche and voice change as indicators of the pubertal growth spurt, Acta Odontol. Scand. 1980; 38: 170 - 86.

5. Hägg U. Taranger J. Skeletal stages of the hand and wrist as indicators of the pubertal growth spurt, Acta Odontol. Scand. 1980; 38: 187 - 200.

6. Hägg U. Taranger J. Dental emergence stages and the pubertal growth spurt, Acta Odontol. Scand. 1981; 39: 295- 306.

7. Taranger J. Hägg U. The timing and duration of adolescent growth, Acta Odontol. Scand. 1980; 38: 57 - 67.

8. Bergersen E. The male adolescent facial growth spurt: its prediction an relation to skeletal maturation. Angle Orthod 1972; 42(4): 319 - 38.

9. Interlandi S. Ortodontia, Bases para a iniciacâo. Ed. Artes Medicas $3^{\text {ra }}$ Edición, Cap 19, 1994.

10. Tiberio. S. 0 estudio de maturacao esquelètica de criancas brasileiras leucodermas, de 8 a 15 años, em referencia a ossificacao dos ossos pisiforme, ganchoso, falanges media e proximal dos dedos 2 e 3. Instituto Metodista de Ensino Superior Sao Bernado Do Campo; 1988.

11. Pancherz H. Hagg V. Dentofacial orthopedic in relation to somatic maturation: An analysis of 7 consecutive cases treated with the Herbst appliance Am J Orthod 1985; 88: 273 - 87.

12. Kopecky G. Fishman L. Timing of cervical headgear treatment based on skeletal maturation Am J Orthod Dentofac Orthop 1993; 104: 162 - 9.

13. Bambha J. Longitudinal cephalometric radiographic study of face and cranium in relation to body height $\mathrm{J}$ Am Dent Assoc 1961; 63: 776 - 99.

14. Nanda R. The rate of growth of several facial components measuered from serial cefalometric roentgenograms Am. J. Ortod 1955; 41: 129 141.

15. Björk A., Helm S. Predition of age of maximum puberal growth in body height Angle Orthod 1967; 37(2): $134-43$.
16. Sierra A. Assessment of dental and skeletal maturity, A new approach Angle Orthod 1987; 57(3): 194 - 208.

17. Coutinho S. Bushgang P. Relationships between mandibular canine calcification stages and skeletal maturity Am J Orthod Dentofac Orthop 1993; 104(3): 262 - 8.

18. Nolla C. Development of the permanent teeth J Dent Child 1960; 27(4): 254-63.

19. Chertkow S. Fatti P. The relationship between tooth mineralization and early radiographic evidence of the ulnar sesamoid Angle Orthod 1979; 49(4): 282 - 8.

20. Chertkow S. Tooth mineralization as an indicator of the puberal growth spurt Am J Orthod 1980; 77(1): 79 91.

21. Gupta S. Assessment of puberty growth spurt in boys and girls: a dental radiographic method J Indian Soc Pedod Prev Dent 1995; 13(1):4 9.

22. Demirjian A. A new system of dental age assesment Human Biol 1973; 45(2): 211 - 27.

23. Manson - Hing L. Vision and oral roentgenology Oral Surg Oral Med Oral Pathol 1962; 15: 173 - 83.

24. Meredith H. Relation between the eruption of selected mandibular permanent teeth and the circumpuberal acceleration in stature J Dent Child 1959; 26: $75-9$.

25. Lewis A. The relationship between tooth formation and other maturational factors Angle Orthod 1960; 30: 70 - 7 .

26. Van Wagenen G Effect of testosterone proportionate on permanent tooth eruption in the monkey (Macaca mulatta) Proc Soc Exp Biol 1950; 73: 296 - 7.

27. Thompson G. Sexual dimorphism in dental mineralization Growth 1975; 39: 289 - 301.

28. Demirjian A. Sexual differences in dental development and prediction of emergence J Dent Res 1980; 59: 1110-22.

\section{Dirección de correspondencia}

Renzo Valverde Montalva

Av. Honorio Delgado 430. Lima 31 - Perú. 381-1950

renzovm@yahoo.com 